\title{
TI

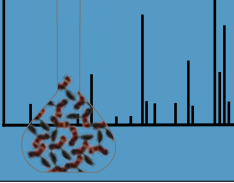 \\ Rapid and Straightforward Quantification of Recombinant Proteins Using Fluorescence Polarization
}

\section{Highlights of Analytical Sciences in Switzerland}

Enrico Condemia, Denis Prim ${ }^{\mathrm{a}}$, Rebecca Brönnimann ${ }^{\mathrm{b}}$, Jonathan Fusco ${ }^{a}$, Simon Creliera, Olimpia Mamula Steiner ${ }^{\mathrm{b}}$, and Jean-Manuel Segura*a

*Correspondence:Prof. Dr. J.-M. Segura, E-mail: jmanuel.segura@hevs.ch; alnstitute of Life Technologies, University of Applied Sciences and Arts Western Switzerland Valais, Route du Rawyl 64, CH-1950 Sion; ' Institute of Chemical Technology, School of Engineering and Architecture, University of Applied Sciences and Arts Western Switzerland Fribourg, Bd de Pérolles 80, $\mathrm{CH}-1705$ Fribourg

\section{Keywords: Antibodies · Assays · Biotechnology ·}

\section{Fluorescence $\cdot$ Recombinant proteins}

Biotechnology enables the production and commercialization of recombinant proteins for diagnostic, therapeutic and research applications. For instance, monoclonal antibodies nowadays represent a fast growing market that accounts for almost half of the biopharmaceutical compounds business with $\$ 75$ billion turnover in 2013.

Quantification of proteins is an important task in order to optimize cell culture conditions and ensure that every step of the production process is in line with the specifications. For therapeutics, precise and reliable dosage is mandatory to avoid adverse effects for the patients. Current analytical techniques such as ELISA or HPLC are time-consuming and often require purification prior to analysis. In this context, there is a strong demand for

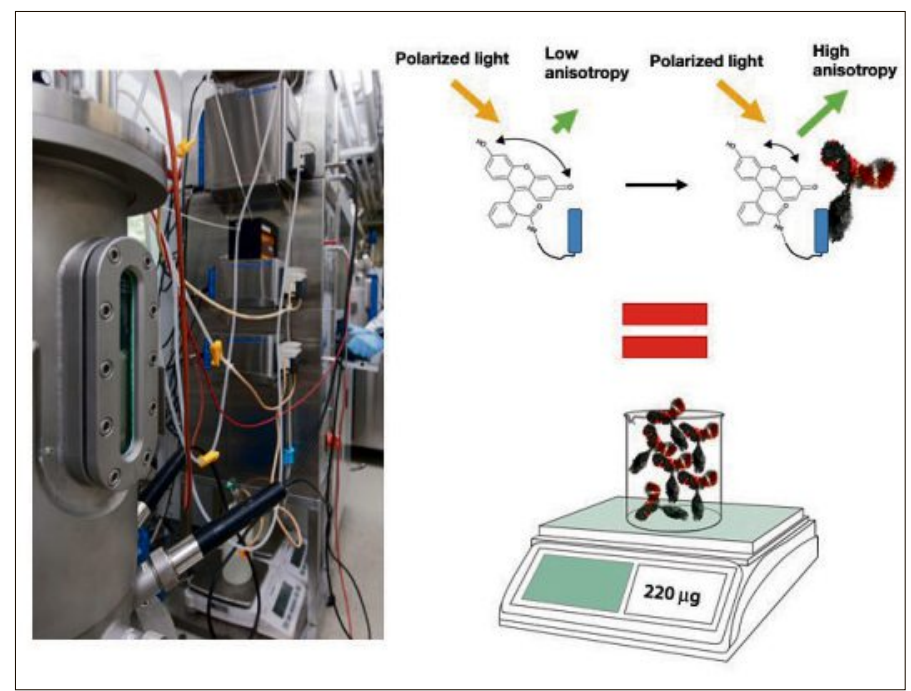

Fluorescence anisotropy of a small specific fluorescent ligand increased upon binding to its recombinant target protein, e.g. a monoclonal antibody, enabling quantification. The assay did not require prior separation of biotechnological cultures making it as straightforward as weighing using a balance. a technology that would be to protein quantification what a balance is to weight measurement: rapid, precise, accurate, robust, and straightforward.

To this effect, we have developed an assay for the rapid quantification of recombinant proteins. It is based on fluorescence polarization and requires little or no pretreatment of samples.

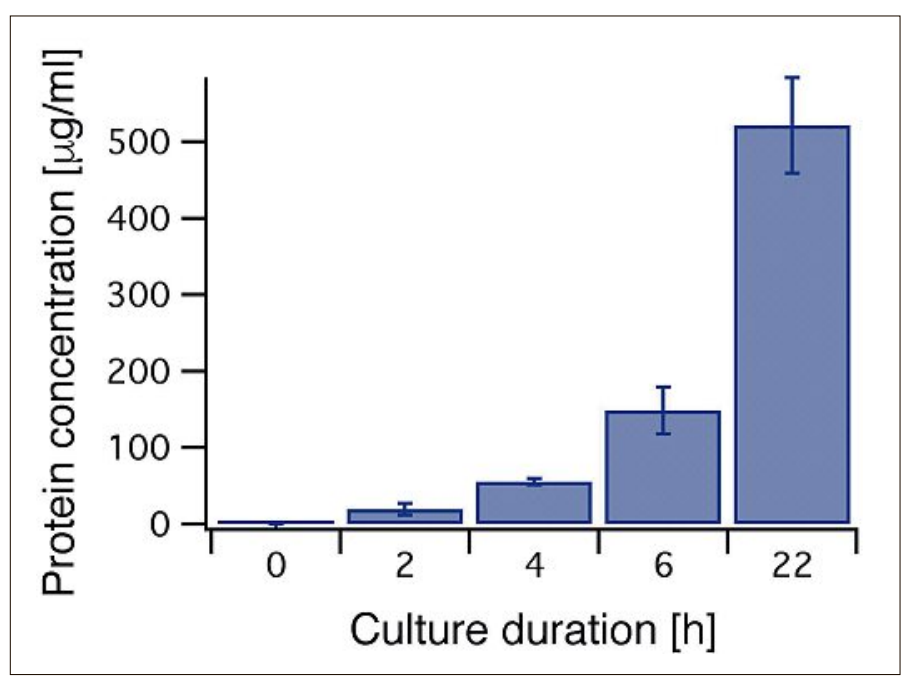

Monitoring of the production of a representative His-tagged protein during E.coli fermentation was obtained by regular sampling and assaying using the fluorescence polarization assay.

The technique makes use of the relationship between the size of a fluorescent molecule and the degree of polarization of its fluorescence when excited using polarized excitation light. Two small fluorescent ligands were designed and synthetized that specifically bind onto two particularly important recombinant protein classes, antibodies and His-tagged proteins. When these small ligands bound to their large recombinant target protein, a concomitant increase in fluorescence polarization anisotropy was recorded and enabled quantification. The assay did not require prior purification: For antibodies it was performed directly in the culture medium, while for His-tagged proteins it was done after the lysis of the bacterial cells. The measurement only required mixing of the specific ligand with typically $100 \mu \mathrm{L}$ of cell culture in 96-well format, and enabled the concentration in target protein to be assessed within less than 15 min using fluorescence readers. Production of prototypical recombinant proteins could be monitored by regular sampling of the culture medium.

Rapid quantification using fluorescence polarization could be a key tool in the future for the efficient optimization and monitoring of bioprocesses.

Received: July 27, 2016

References

D. M. Ecker, S. D. Jones, H. L. Levine, $m A b s$ 2015, 7, 9.

D. M. Jameson, J. A. Ross, Chem. Rev. 2010, 110, 2685. 